\title{
openheart Bleeding after percutaneous coronary intervention: can we still ignore the obvious?
}

\author{
Mony Shuvy, ${ }^{1}$ Dennis T Ko ${ }^{1,2,3}$
}

To cite: Shuvy M, Ko DT. Bleeding after percutaneous coronary intervention: can we still ignore the obvious? Open Heart 2014;1:e000036. doi:10.1136/openhrt-2014000036

Accepted 6 February 2014

\section{SLinked}

- http://dx.doi.org/10.1136/ openhrt-2013-000021

\section{CrossMark}

\footnotetext{
${ }^{1}$ Schulich Heart Centre Centre, Toronto, Ontario, Canada

2Department of Medicine, University of Toronto,

Toronto, Ontario, Canada ${ }^{3}$ Institute for Clinical Ontario, Canada

Correspondence to Dr Dennis T Ko; dennis.ko@ices.on.ca
} Sunnybrook Health Sciences Evaluative Sciences, Toronto,
Percutaneous coronary intervention (PCI) is the most common cardiac invasive procedure to treat patients with coronary artery disease. In the USA, it is estimated that more than one million patients undergo PCI each year. Fearsome complications such as coronary dissection or acute vessel closure in the balloon angioplasty era have been largely mitigated with the introduction of coronary artery stents and the routine use of antiplatelet and antithrombotic therapy. In the contemporary practice of interventional cardiology, it is recognised that bleeding has become the most common early complication associated with PCI. While the incidence of bleeding varies across studies, recent data from the USA found major bleeding occurs at a rate of $1.7 \%$ after PCI, ${ }^{1}$ about half from the site of arterial access and half from non-access locations, most commonly the gastrointestinal (GI) tract. ${ }^{1}$

As a practising clinician, it is easy to remember situations where a patient has undergone successful PCI and then suffered a major bleeding episode. Treating patients who bled after PCI is often complicated because one needs to consider the appropriate intervention to manage the active bleeding, to weigh the potential benefits and risks of withholding or withdrawing antiplatelet or anticoagulation therapy and to evaluate whether red blood cell transfusion outweighs its potential adverse effects. Indeed, many factors directly relating to the bleeding itself and its management have been implicated to be associated with worse outcomes among patients who experienced bleeding after PCI procedures. $^{2}$

In this issue of Open Heart, Kwok et a $\vec{l}^{3}$ make an important contribution to this field, by examining the relationship between bleeding complications in PCI and subsequent death. This comprehensive systematic review is one of the largest to date, with 38 published studies, and more than 500000 patients. Their work reveals several important insights. First, the authors found that major bleeding with PCI is associated with more than threefold increase in death or major adverse cardiac outcome. This increased risk was sustained even after 12 months after the procedure, suggesting the long-lasting adverse impact of the bleeding event. Second, although it has been suggested that the adverse impact of bleeding may simply be a surrogate for patients with multiple comorbidities, the authors found increased risk associated with PCI bleeding even after consideration of confounding factors, suggesting that bleeding is independently linked to mortality and adverse cardiac outcomes after PCI. Finally, the authors found that the strength of association between PCI bleeding with death varied significantly according to the definition of bleeding used in the individual studies. This information could be particularly helpful for quality improvement initiatives to select a bleeding definition that maximises detection, while at the same time that is also meaningful to predict adverse outcomes. To complement these data, Marso and colleagues conducted an analysis that included more than three million PCI procedures in the USA and found that $12.1 \%$ of all in-hospital deaths after PCI are attributed to bleeding episodes. ${ }^{1}$ Furthermore, they demonstrated that bleeding was harmful in patients at different bleeding risks. Given the importance of bleeding after PCI, several aspects that may reduce the risk of bleeding should be considered in all patients. ${ }^{1}$

\section{ARTERIAL ACCESS SITE}

The radial artery is a superficial artery that is readily compressible compared with the femoral artery, which is in a much deeper location and with a harder to achieve haemostasis. Accordingly, performing PCI via the 
radial artery is currently the most effective way to reduce access site bleeding. In the RadIal Vs femorAL access for coronary intervention (RIVAL) trial, investigators randomised 7032 patients with acute coronary syndrome (ACS) who were undergoing invasive evaluation with coronary angiography. ${ }^{4}$ The primary outcome was a composite of death, myocardial infarction, stroke or non-coronary artery bypass graft-related major bleeding at 30 days. The hazard of bleeding was significantly reduced by more than half in patients randomised to radial access. At 30 days, $3 \%$ in the femoral group had large haematoma and $0.6 \%$ had pseudoaneurysm requiring closure vs $1.2 \%$ with haematoma and $0.2 \%$ with pseudoaneurysm in the radial group. ${ }^{4}$ Performing radial access during ACS may also lead to improved clinical outcomes. Although the primary outcome of RIVAL was not significantly different in the treatment groups, evidence of a benefit for patients with ST-segment elevation myocardial infarction (STEMI) has emerged. Among the 1958 patients with STEMI enrolled in the study, the rate of primary outcome was $3.1 \%$ in the radial group vs $5.2 \%$ in the femoral group and a $40 \%$ lower hazard of outcome was seen. ${ }^{5}$ Despite these data, the adoption continues to be very low in many interventional centres.

\section{APPROPRIATE ANTIPLATELET AND ANTITHROMBOTIC THERAPY}

Dual antiplatelet therapy with aspirin and clopidogrel has been the mainstay treatment for the majority of patients with ACS and those undergoing PCI procedures. Recently, more potent antiplatelet drugs such as prasugrel and ticagrelor have been shown to be associated with additional benefit compared with clopidogrel in the management of patients with ACS. However, both medications are associated with an increased risk of bleeding. ${ }^{6}$ For prasugrel, it is important to note that there was no net clinical benefit for patients of 75 years or older or patients weighing less than $60 \mathrm{~kg}$, and harm was observed for patients who had a previous stroke or transient ischaemic attack. ${ }^{7}$ Furthermore, trials of newer antiplatelet agents were performed in patients with ACS and not for patients with stable coronary disease undergoing PCI.

Evidence continues to evolve regarding the optimal duration for dual antiplatelet therapy after PCI. Practice guidelines from the USA currently recommend 12 months of dual antiplatelet therapy for ACS and drug-eluting stents implementation and a minimum of 1 month but ideally 12 months for bare metal stents. ${ }^{8}$ Another clinical dilemma is the optimal antiplatelet regimen in patients who require long-term anticoagulation therapy which is associated with substantial risk of bleeding and should be minimised if possible. ${ }^{2}$ Post PCI GI bleeding is a common problem, in particular, in patients with ACS.

For antithrombotic therapy with PCI, there is also a great complexity because of the vast number of choices that include heparin, heparin and glycoprotein IIb/IIIa inhibitors, low molecular weight heparin, fondaparinux, bivalirudin and others. In the Acute Catheterisation and Urgent Intervention Triage Strategy (ACUITY) trial, 13819 patients with ACS were randomised and demonstrated that bivalirudin was associated with an approximately $50 \%$ reduction in bleeding complications compared with the glycoprotein IIb/IIIa group, with similar ischaemic adverse events. ${ }^{9}$

The appropriate use of antiplatelet and antithrombotic therapy should be individualised because it requires careful consideration to balance the risks of bleeding from these agents with their potential benefits depending on the clinical presentations. Factors that have been consistently shown to be associated with higher risk of PCI-associated bleeding include older age, female sex, body mass index at the extremes (overweight or underweight), acute presentation (STEMI, shock, cardiac arrest) and medical comorbidities (renal failure, prior bleeding, anaemia). ${ }^{2}{ }^{10}$ Investigators have also developed bleeding risk scores to predict chance of bleeding risk PCI, which can be used at the beside to facilitate discussion of risk of bleeding with patients and potentially assist in treatment decisions. ${ }^{1011}$

\section{VASCULAR CLOSURE DEVICES}

Although vascular closure devices are sometimes considered as a bleeding reduction strategy for patients with femoral access, early studies comparing vascular closure devices and manual compression have suggested an overall higher risk of vascular complication. ${ }^{8}$ In contrast, recent larger registries have suggested that the use of vascular closure devices is associated with lower risk of bleeding and vascular complications. It is difficult to provide a conclusive statement. In addition, it is difficult to distil the literature on vascular closure device because they have evolved substantially over time and few conclusive randomised studies are available. Accordingly, practice guidelines recommend vascular closure devices for early haemostasis and faster ambulation, but do not recommend for the purpose of decreasing vascular complications, including bleeding. ${ }^{8}$

In summary, in view of the wealth of evidence demonstrating the adverse effect of bleeding after PCI, it is time that clinicians become proactive to minimise the chance of bleeding after PCI by adopting the latest evidence in clinical practice to improve the outcome of our patients.

Funding DTK is supported by a Clinician Scientist Award from the Heart and Stroke Foundation of Canada, Ontario provincial office.

Competing interests None.

Provenance and peer review Commissioned; internally peer reviewed.

Open Access This is an Open Access article distributed in accordance with the Creative Commons Attribution Non Commercial (CC BY-NC 3.0) license, which permits others to distribute, remix, adapt, build upon this work noncommercially, and license their derivative works on different terms, provided the original work is properly cited and the use is non-commercial. See: http:// creativecommons.org/licenses/by-nc/3.0/ 


\section{REFERENCES}

1. Chhatriwalla AK, Amin AP, Kennedy KF, et al. Association between bleeding events and in-hospital mortality after percutaneous coronary intervention. JAMA 2013;309:1022-9.

2. Ko DT, Yun L, Wijeysundera HC, et al. Incidence, predictors, and prognostic implications of hospitalization for late bleeding after percutaneous coronary intervention for patients older than 65 years. Circ Cardiovasc Interv 2010;3:140-7.

3. Kwok CS, Rao SV, Myint PK, et al. Major bleeding after percutaneous coronary intervention and risk of subsequent mortality: a systematic review and meta-analysis. Open Heart 2014;1:1 e000021.

4. Jolly SS, Yusuf S, Cairns J, et al. Radial versus femoral access for coronary angiography and intervention in patients with acute coronary syndromes (RIVAL): a randomised, parallel group, multicentre trial. Lancet 2011;377:1409-20.

5. Mehta SR, Jolly SS, Cairns J, et al. Effects of radial versus femoral artery access in patients with acute coronary syndromes with or without ST-segment elevation. J Am Coll Cardiol 2012;60:2490-9.

6. Capodanno D, Ferreiro JL, Angiolillo DJ. Antiplatelet therapy: new pharmacological agents and changing paradigms. J Thromb Haemost 2013;11:316-29.

7. Wiviott SD, Braunwald E, McCabe $\mathrm{CH}$, et al. Prasugrel versus clopidogrel in patients with acute coronary syndromes. $N$ Engl J Med 2007;357:2001-15.
8. Levine GN, Bates ER, Blankenship JC, et al. 2011 ACCF/AHA/SCAI Guideline for percutaneous coronary intervention. A report of the American College of Cardiology Foundation/American Heart Association Task Force on Practice Guidelines and the Society for Cardiovascular Angiography and Interventions. J Am Coll Cardiol 2011;58:e44-122.

9. Stone GW, McLaurin BT, Cox DA, et al. Bivalirudin for patients with acute coronary syndromes. N Engl J Med 2006;355: 2203-16.

10. Rao SV, McCoy LA, Spertus JA, et al. An updated bleeding model to predict the risk of post-procedure bleeding among patients undergoing percutaneous coronary intervention. A report using an expanded bleeding definition from the National Cardiovascular Data Registry CathPCI Registry. JACC Cardiovasc Interv 2013;6: 897-904.

11. Mehran R, Pocock S, Nikolsky E, et al. Impact of bleeding on mortality after percutaneous coronary intervention results from a patient-level pooled analysis of the REPLACE-2 (randomized evaluation of $\mathrm{PCl}$ linking angiomax to reduced clinical events), ACUITY (acute catheterization and urgent intervention triage strategy), and HORIZONS-AMI (harmonizing outcomes with revascularization and stents in acute myocardial infarction) trials. JACC Cardiovasc Interv 2011;4: 654-64. 